\title{
Dose-dependent effects of glucocorticoids on pulmonary vascular development in a murine model of hyperoxic lung injury
}

\author{
Marta Perez ${ }^{1}$, Kamila Wisniewska', Keng Jin Lee ${ }^{1}$, Herminio J. Cardona' ${ }^{1}$, Joann M. Taylor ${ }^{1}$ and Kathryn N. Farrow ${ }^{1}$
}

BACKGROUND: Exposure of neonatal mice to hyperoxia results in pulmonary vascular remodeling and aberrant phosphodiesterase type 5 (PDE5) signaling. Although glucocorticoids are frequently utilized in the NICU, little is known about their effects on the developing pulmonary vasculature and on PDE5. We sought to determine the effects of hydrocortisone $(\mathrm{HC})$ on pulmonary vascular development and on PDE5 in a neonatal mouse model of hyperoxic lung injury.

METHODS: C57BL/ 6 mice were placed in $21 \% \mathrm{O}_{2}$ or $75 \% \mathrm{O}_{2}$ within $24 \mathrm{~h}$ of birth and received $\mathrm{HC}(1,5$, or $10 \mathrm{mg} / \mathrm{kg}$ subcutaneously every other day) or vehicle. At $14 \mathrm{~d}$, right ventricular hypertrophy (RVH), medial wall thickness (MWT), lung morphometry, and pulmonary artery (PA) PDE5 activity were assessed. PDE5 activity was measured in isolated pulmonary artery smooth muscle cells exposed to 21 or $95 \% \mathrm{O}_{2} \pm 100$ $\mathrm{nmol} / \mathrm{l} \mathrm{HC}$ for $24 \mathrm{~h}$.

RESULTS: Hyperoxia resulted in alveolar simplification, RVH, increased MWT, and increased PA PDE5 activity. HC decreased hyperoxia-induced RVH and attenuated MWT. HC had dose-dependent effects on alveolar simplification. HC decreased hyperoxia-induced PDE5 activity both in vivo and in vitro.

CONCLUSIONS: $\mathrm{HC}$ decreases hyperoxia-induced pulmonary vascular remodeling and attenuates PDE5 activity. These findings suggest that $\mathrm{HC}$ may protect against hyperoxic injury in the developing pulmonary vasculature.

B ronchopulmonary dysplasia (BPD) remains one of the most common complications of prematurity. Despite advances in neonatal care and increased survival of infants born extremely premature, the incidence of BPD has remained relatively unchanged (1). Anywhere from 22 to $68 \%$ of infants born prematurely will go on to develop BPD, increasing mortality, morbidity, and health care costs (1-3). One well-known complication of BPD is the development of pulmonary hypertension $(\mathrm{PH})$. Of those infants with moderate to severe BPD, up to $25 \%$, will progress to develop $\mathrm{PH}(4-7)$. The outcomes for infants with $\mathrm{BPD}$-associated $\mathrm{PH}$ are significantly worse when compared with infants with BPD alone $(6,8)$. BPD infants with $\mathrm{PH}$ are at risk for development of further cardiovascular complications, including right ventricular hypertrophy (RVH) and right heart failure. The best treatment options for BPDassociated $\mathrm{PH}$ remain unknown.

In order to study the pulmonary and vascular processes underlying BPD, we utilized the murine model of hyperoxic lung injury. The murine lung at the time of birth is histologically similar to the lung of a premature human infant born at $24-28$ wk of gestation (9). We have previously reported that 2-wk exposure of neonatal mice to hyperoxia results in pathological changes similar to anomalies seen in human infants with BPD and BPD-associated PH (10), including development of RVH, remodeling of small pulmonary arteries, and alveolar simplification (10-12). An additional finding in the murine model of hyperoxic lung injury is increased activation of phosphodiesterase type 5 (PDE5) in the small pulmonary arteries (10). PDE5 is the major phosphodiesterase isoform in lung vasculature that catalyzes the conversion of cyclic guanosine monophosphate to its inactive form guanosine 59-monophosphate (GMP), ultimately resulting in vasoconstriction. Although sildenafil, a PDE5 inhibitor, is sometimes used in the treatment of infants with $\mathrm{BPD}$-associated $\mathrm{PH}$, its use in those patients is not well studied $(13,14)$. Additionally, sildenafil now comes with a black box warning against its use in children, further underscoring the need for additional neonatal $\mathrm{PH}$ therapies (15).

Glucocorticoids have been historically used in neonatal care for the treatment of pressor-resistant hypotension and adrenal insufficiency, as well as for BPD treatment and prophylaxis. Although these medications continue to be used with some frequency in the NICU (16), their effects on neonatal pulmonary vasculature remain largely unknown. Glucocorticoids have been reported to attenuate $\mathrm{PH}$ in meconium aspiration syndrome, a disease with a strong inflammatory component $(17,18)$. Prenatal glucocorticoids have been found to improve postnatal transition in lambs with persistent pulmonary hypertension of the newborn (19). Our lab has previously demonstrated that postnatal hydrocortisone (HC) improves oxygenation, attenuates oxidative stress, and decreases hyperoxia-induced PDE5 activation in a sheep model of persistent

'Department of Pediatrics, Northwestern University, Chicago, Illinois. Correspondence: Marta Perez (mtperez@luriechildrens.org)

Received 29 May 2015; accepted 31 October 2015; advance online publication 3 February 2016. doi:10.1038/pr.2016.1 


\section{Articles $\mid$ Perezetal.}

pulmonary hypertension of the newborn (20). In addition, HC treatment of isolated pulmonary artery smooth muscle cells (PASMC) attenuated PDE5 activity induced by $24-\mathrm{h}$ hyperoxia exposure (21). In the current study, we set out to determine the effects of $\mathrm{HC}$ on pulmonary vasculature, lung architecture, and vascular PDE5 activity in a mouse model of hyperoxic lung injury, which more closely approximates the preterm pulmonary vasculature.

\section{RESULTS}

\section{HC Attenuates Hyperoxia-Induced RVH and Ameliorates Remodeling of Small PAs in Neonatal Mice}

As previously described (10), hyperoxia mice demonstrated RVH as measured by the Fulton's index ( $125 \pm 4 \%$ vs. normoxia vehicle) (Figure 1). Treatment with all doses of $\mathrm{HC}$ led to significant decreases in Fulton's index $(90 \pm 3,83 \pm 7$, and $87 \pm 3 \%$ for 1,5 , and $10 \mathrm{mg} / \mathrm{kg}$, respectively, vs. hyperoxia-vehicle mice) (Figure 1). Interestingly, treatment with 5 and $10 \mathrm{mg} / \mathrm{kg} \mathrm{HC}$ resulted in a small but statistically significant increase in $\mathrm{RVH}$ in normoxia when compared with vehicle-treated animals (117 \pm 4 and $113 \pm 8 \%$, respectively, vs. normoxia vehicle).

In addition to development of $\mathrm{RVH}$ and consistent with our previously published findings (10), hyperoxia was associated with a modest but significant increase in medial wall thickness $(119 \pm 10 \%$ vs. normoxia vehicle) as demonstrated in Figure 2a. This was significantly attenuated by the 1 and $5 \mathrm{mg} /$ $\mathrm{kg}$ doses of $\mathrm{HC}(73 \pm 3,84 \pm 5 \%$ vs. hyperoxia-vehicle mice) as shown in Figure 2a. Representative vessel images are shown in Figure 2b.

\section{Treatment with Higher HC Doses Has Adverse Effects on Alveolar Growth in Hyperoxia-Exposed Mice}

To evaluate the effects of oxygen and HC exposure on alveolar growth, mean alveolar area and mean chord length were measured in hematoxylin-stained lung sections. Similar to previous reports $(10,22)$, hyperoxia exposure increased both mean

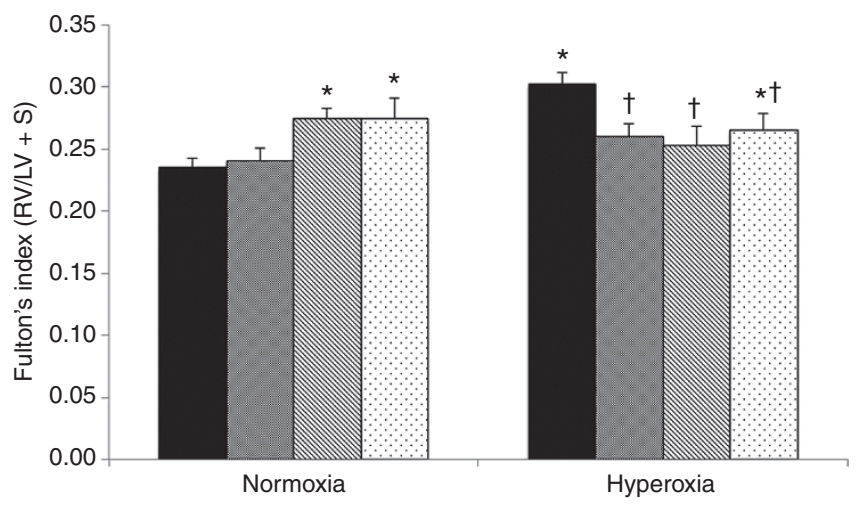

Figure 1. Hydrocortisone attenuates RVH induced by $14 \mathrm{~d}$ of hyperoxia exposure in neonatal mice. Mice exposed to $75 \% \mathrm{O}_{2}$ (hyperoxia) or room air (normoxia) \pm hydrocortisone $(1,5$, or $10 \mathrm{mg} \mathrm{HC})$ or sterile water (vehicle) were killed and hearts were dissected ( $n=4-20$ per group; black columns: vehicle, gray: $1 \mathrm{mg} \mathrm{HC}$, hatched: $5 \mathrm{mg} \mathrm{HC}$, dotted: $10 \mathrm{mg} \mathrm{HC}$ ). Fulton's index was measured as the weight of the right ventricle (RV) divided by the weight of the left ventricle plus septum $(L V+S)$. Data are shown as mean \pm SEM, ${ }^{*} P<0.05$ vs. normoxia vehicle, ${ }^{\dagger} P<0.05$ vs. hyperoxia vehicle. $\mathrm{RVH}$, right ventricular hypertrophy; $\mathrm{HC}$, hydrocortisone. alveolar area and mean chord length $(137 \pm 3$ and $128 \pm 4 \%$, respectively, vs. normoxia vehicle) (Figure 3). Alveolar simplification was worsened in hyperoxic animals treated with $10 \mathrm{mg} / \mathrm{kg} \mathrm{HC}$ but was not significantly affected by lower HC doses (Figures 3 and 4). Of note, $10 \mathrm{mg} / \mathrm{kg} \mathrm{HC}$ dose exposure in normoxic mice resulted in statistically significant increases in mean alveolar area and mean chord length as demonstrated in Figure 3a, b $(127 \pm 10$ and $111 \pm 3 \%$, respectively, vs. normoxia vehicle). Representative lung sections are shown in Figure 4. Furthermore, we investigated the effects of steroid treatment on elastin, an important mediator of alveolar development, but did not observe any significant differences in parenchymal elastin content in HC-treated animals (Figure 5).

\section{Effects of HC Treatment on Body Weight Are Variable and Dose Dependent.}

Given the well-described association between poor somatic growth and both prolonged oxygen exposure and glucocorticoid treatment in rodents $(23,24)$, we sought to determine the effects of oxygen and steroid exposure on body weight in neonatal mice in our model. In the current study, we did not find

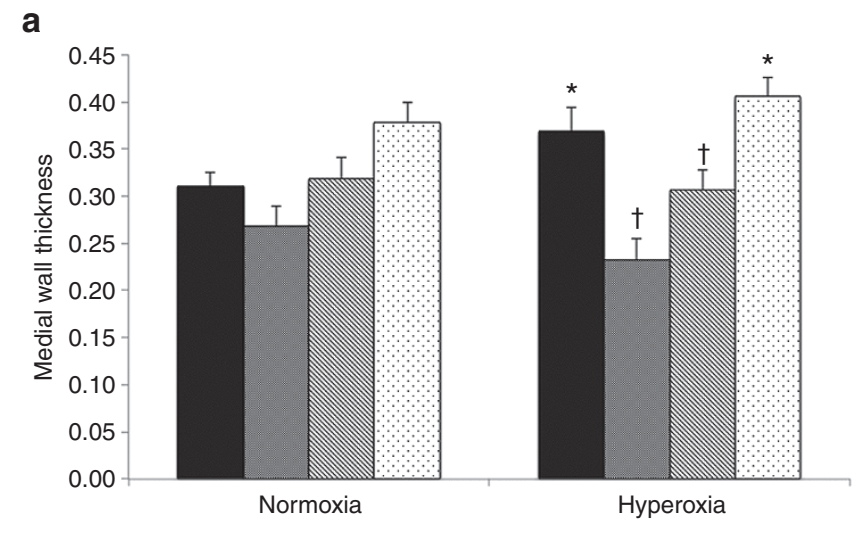

b
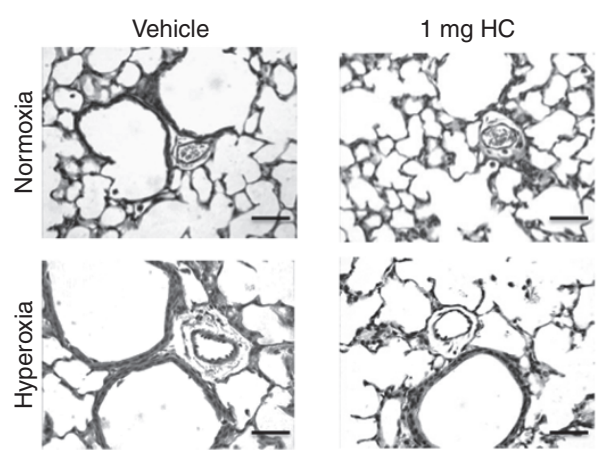

Figure 2. Hydrocortisone ameliorates remodeling of small pulmonary arteries in a dose-dependent manner in hyperoxia-exposed neonatal mice. Mice exposed to $75 \% \mathrm{O}_{2}$ (hyperoxia) or room air (normoxia) \pm hydrocortisone $(1,5$, or $10 \mathrm{mg} \mathrm{HC}$ ) or sterile water (vehicle) were killed and lungs were inflation fixed ( $n=5-16$ per group). (a) Pulmonary hypertension was assessed by medial wall thickness (MWT) of small PAs (black columns: vehicle, gray: $1 \mathrm{mg} \mathrm{HC}$, hatched: $5 \mathrm{mg} \mathrm{HC}$, dotted: $10 \mathrm{mg} \mathrm{HC}$ ). (b) Representative H\&E-stained lung sections (magnification 40x) for normoxia vehicle, normoxia $+1 \mathrm{mg} \mathrm{HC}$, hyperoxia vehicle, and hyperoxia $+1 \mathrm{mg} \mathrm{HC}$ mice. Data are shown as mean $\pm \mathrm{SEM}, * P<0.05$ vs. normoxia vehicle, ${ }^{\dagger} P<0.05$ vs. hyperoxia vehicle. $B a r=50 \mu \mathrm{m} . \mathrm{HC}$, hydrocortisone. 


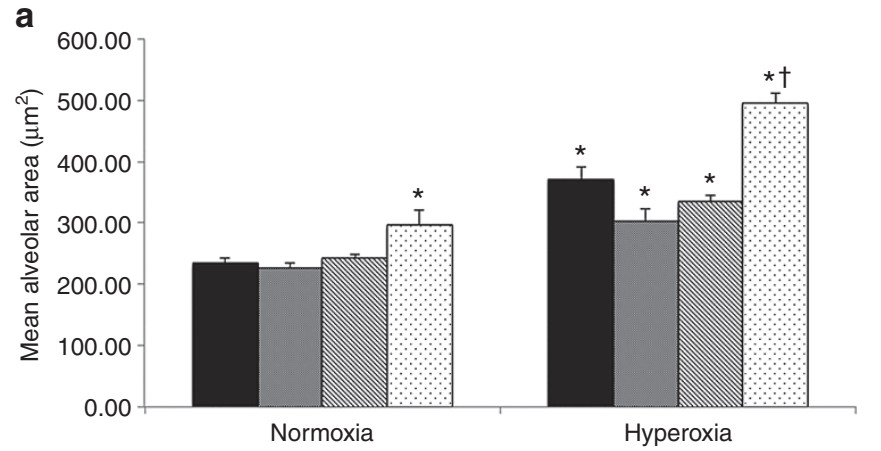

b

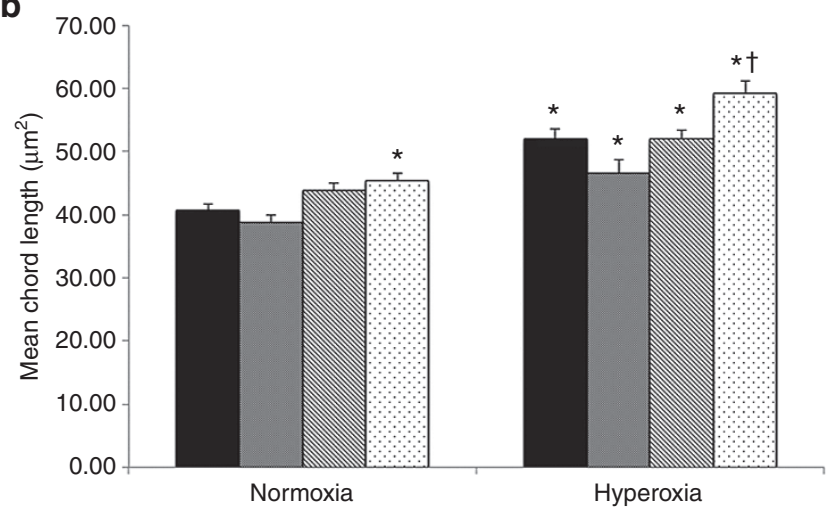

Figure 3. Hydrocortisone effects on alveolar simplification are dose dependent. Mice exposed to $75 \% \mathrm{O}_{2}$ (hyperoxia) or room air (normoxia) \pm hydrocortisone $(1 \mathrm{mg} \mathrm{HC}, 5 \mathrm{mg} \mathrm{HC}$, or $10 \mathrm{mg} \mathrm{HC}$ ) or sterile water (vehicle) were killed and lungs were inflation fixed ( $n=5-17$ per group). Hematoxylin-stained sections were imaged at 20x, and (a) mean alveolar area (black columns: vehicle, gray: $1 \mathrm{mg} \mathrm{HC}$, hatched: $5 \mathrm{mg} \mathrm{HC}$, dotted: $10 \mathrm{mg} \mathrm{HC}$ ) and (b) mean chord length (black columns: vehicle, gray: $1 \mathrm{mg}$ $\mathrm{HC}$, hatched: $5 \mathrm{mg} \mathrm{HC}$, dotted: $10 \mathrm{mg} \mathrm{HC}$ ) were measured using Scion software. Data are shown as mean $\pm \mathrm{SEM},{ }^{*} P<0.05$ vs. normoxia vehicle, ${ }^{\dagger} P<0.05$ vs. hyperoxia vehicle. $\mathrm{HC}$, hydrocortisone.
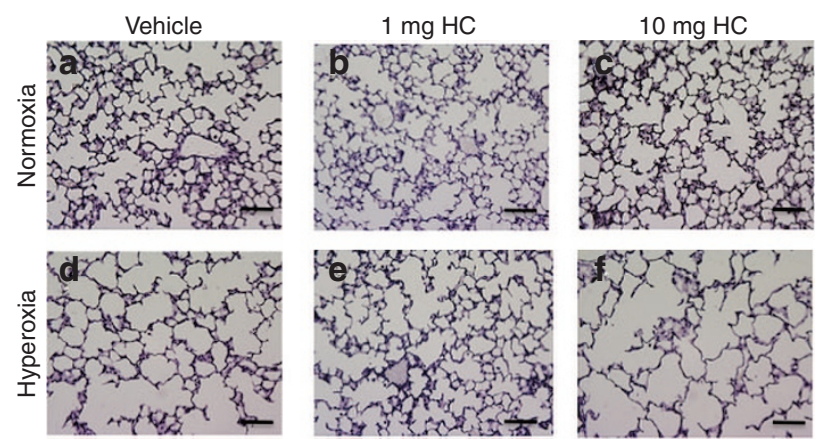

Figure 4. Hyperoxia-induced alveolar simplification is worsened by high dose hydrocortisone but is not affected by lower dose hydrocortisone. Mice exposed to $75 \% \mathrm{O}_{2}$ (hyperoxia) or room air (normoxia) \pm hydrocortisone $(1,5$, or $10 \mathrm{mg} \mathrm{HC}$ ) or sterile water (vehicle) were killed and lungs were inflation fixed. Hematoxylin-stained sections were imaged at 20x. Representative hematoxylin-stained lung sections are shown for normoxia vehicle (a), normoxia $+1 \mathrm{mg} \mathrm{HC}(\mathbf{b})$, normoxia $+10 \mathrm{mg} \mathrm{HC}(\mathbf{c})$, hyperoxia vehicle (d), hyperoxia $+1 \mathrm{mg} \mathrm{HC}(\mathbf{e})$, and hyperoxia $+10 \mathrm{mg} \mathrm{HC}$ mice (f). $\mathrm{Bar}=200 \mu \mathrm{m}$. HC, hydrocortisone.

any significant effects of hyperoxia on body weight (Figure 6). The effects of HC treatment on body weight were variable. The $1 \mathrm{mg} / \mathrm{kg}$ dose of $\mathrm{HC}$ was associated with a statistically a

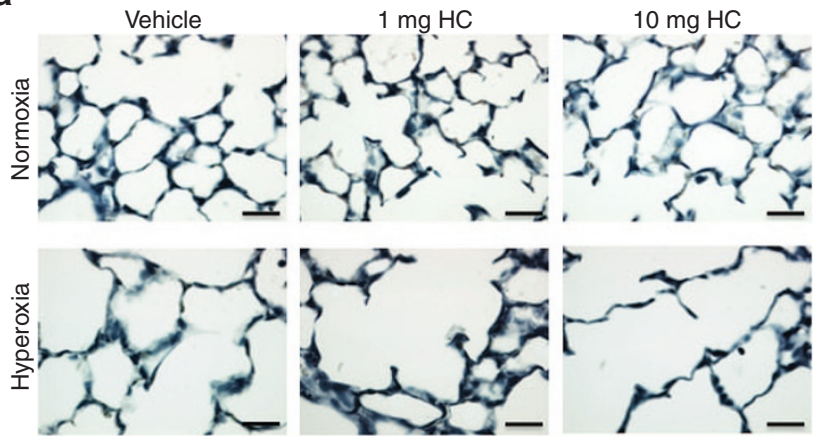

b

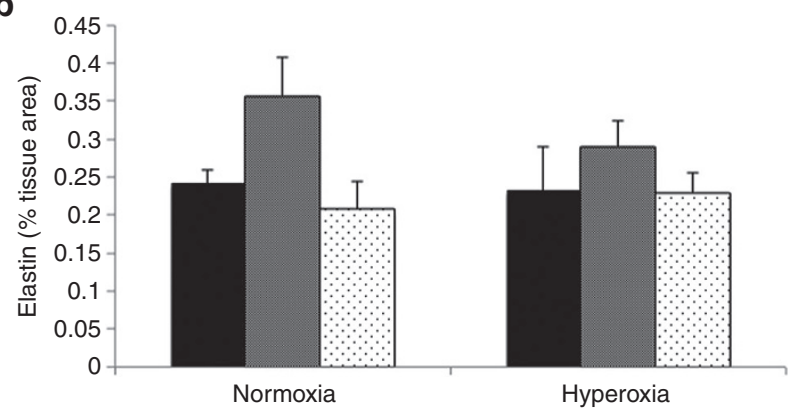

Figure 5. Hydrocortisone does not affect parenchymal elastin content. Mice exposed to $75 \% \mathrm{O}_{2}$ (hyperoxia) or room air (normoxia) \pm hydrocortisone $(1$, or $10 \mathrm{mg} \mathrm{HC}$ ) or sterile water (vehicle) were killed and lungs were inflation fixed. Lung sections were stained with the Verhoeff-van Gieson stain and imaged at 40x. (a) Representative elastin-stained lung sections are shown for normoxia vehicle, normoxia $+1 \mathrm{mg} \mathrm{HC}$, normoxia $+10 \mathrm{mg}$ $\mathrm{HC}$, hyperoxia vehicle, hyperoxia $+1 \mathrm{mg} \mathrm{HC}$, and hyperoxia $+10 \mathrm{mg} \mathrm{HC}$ mice. $B a r=25 \mu \mathrm{m}$. (b) Elastin was calculated as percentage of elastin area/ percentage tissue area. $\mathrm{HC}$, hydrocortisone.

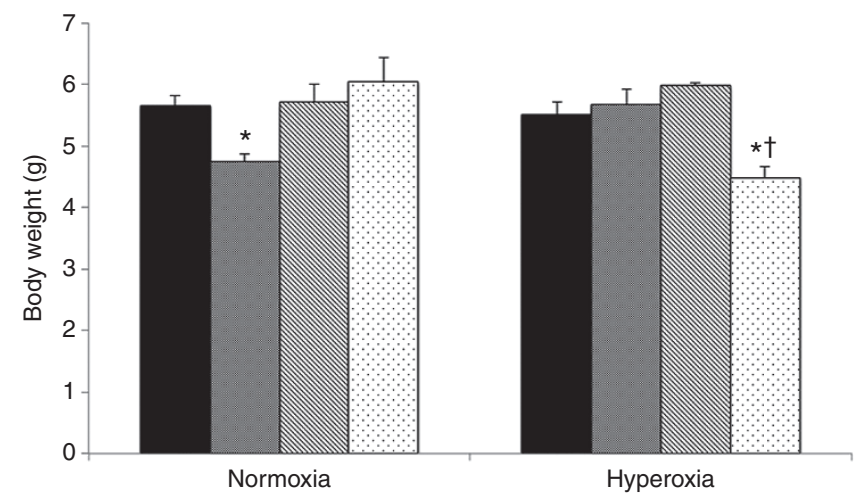

Figure 6. Hyperoxia exposure leads to decreased body weight and this is further reduced by treatment with variable doses of hydrocortisone. Mice exposed to $75 \% \mathrm{O}_{2}$ (hyperoxia) or room air (normoxia) \pm hydrocortisone $(1,5$, or $10 \mathrm{mg} \mathrm{HC})$ or sterile water (vehicle) were killed and body weight was obtained ( $n=7-25$ per group; black columns: vehicle, gray: $1 \mathrm{mg} \mathrm{HC}$, hatched: $5 \mathrm{mg} \mathrm{HC}$, dotted: $10 \mathrm{mg} \mathrm{HC}$ ). Data are shown as mean $\pm \mathrm{SEM}$, ${ }^{*} P<0.05$ vs. normoxia vehicle, ${ }^{\dagger} P<0.05$ vs. hyperoxia vehicle. $\mathrm{HC}$, hydrocortisone.

significant decrease in body weight in normoxic animals $(84 \pm 2 \%$ vs. normoxia vehicle) as shown in Figure 6. Only the $10 \mathrm{mg} / \mathrm{kg}$ dose of $\mathrm{HC}$ resulted in significant decrease in weight in hyperoxia-exposed animals ( $82 \pm 3 \%$ vs. hyperoxia vehicle) (Figure 6). 


\section{Articles $\mid$ Perezetal.}

\section{HC Treatment Attenuates Hyperoxia-Induced Vascular PDE5 Activity in Both Neonatal Mice Pulmonary Artery (PA) and in Isolated Primary PASMC}

To elucidate the potential mechanisms through which $\mathrm{HC}$ attenuates cardiovascular remodeling induced by hyperoxia exposure, we investigated the effects of $\mathrm{HC}$ treatment on vascular PDE5 activity both in vivo and in vitro. Our lab has previously demonstrated that exposure to hyperoxia results in increased PA PDE5 activity in neonatal sheep and mice as well as in isolated fetal sheep PASMC $(10,25,26)$. In agreement with our previous reports, PDE5 activity was upregulated in PAs from hyperoxia-exposed mice $(167 \pm 29 \%$ vs. normoxiavehicle mice) (Figure 7a) (10). Treatment with $1 \mathrm{mg} / \mathrm{kg} \mathrm{HC}$ resulted in significant decreases in vascular PDE5 activity ( $40 \pm 13 \%$ vs. hyperoxia vehicle) (Figure 7a). We then examined the effects of HC on PDE5 activity in isolated mouse PASMC to determine if $\mathrm{HC}$ was acting specifically on the smooth muscle as we have previously described (20,21). A $24 \mathrm{~h}$ exposure of isolated PASMC to $95 \% \mathrm{O}_{2}$ increased PDE5 activity ( $244 \pm 47$ vs. $21 \%$ untreated) (Figure 7 b) while treatment with $100 \mathrm{nmol} / \mathrm{l} \mathrm{HC}$ significantly attenuated that activity ( $34 \pm 10$ vs. $95 \%$ untreated) (Figure 7 b).

Furthermore, we found that the effects of HC on PDE5 activation are rapid, occurring within 90 min of hyperoxia exposure (Figure 8).

\section{DISCUSSION}

BPD is an important cause of long-term morbidity and mortality in formerly premature infants. Development of $\mathrm{PH}$ can complicate the clinical course of BPD, further worsening outcomes $(5,7)$. Optimal treatments for BPD-associated $\mathrm{PH}$ have not been established. While glucocorticoids have been previously utilized in the treatment of $B P D$, very little is currently known about their effects on the developing pulmonary vasculature. We have previously demonstrated that $\mathrm{HC}$ treatment improves oxygenation and normalizes
PDE5 activity in the sheep model of persistent pulmonary hypertension of the newborn (20,21). In the current study, we sought to determine the effects of HC on pulmonary vascular development and on PDE5 signaling in a mouse model of hyperoxic lung injury. We found that HC treatment of neonatal mice decreased markers of $\mathrm{PH}$, namely $\mathrm{RVH}$ and remodeling of small PAs, with dose-dependent effects on alveolar development. In addition, HC treatment normalized vascular PDE5 activity both in vivo and in vitro.

We utilized the murine model of hyperoxic lung injury to approximate the disease seen in infants with BPD. Similar to the previous work from our lab, 14-d exposure of neonatal mice to hyperoxia resulted in significant RVH and remodeling of small PAs compared with mice raised in normoxic conditions (Figures 1 and 2) (10). HC treatment led to significant decreases in $\mathrm{RVH}$ at all doses in hyperoxia-exposed mice (Figure 1). Interestingly, treatment with higher doses of $\mathrm{HC}$ was associated with development of RVH in normoxic animals, an effect that was not seen at the lowest dose (Figure 1). Although long-term, postnatal exposure to glucocorticoids has been associated with left ventricular hypertrophy in formerly premature infants with chronic lung disease, little is known about the effects of these drugs on the developing right ventricle (RV) $(27,28)$. Another study has previously shown trends toward increased right ventricular chamber diameter with dexamethasone treatment of healthy neonatal rats (29). An additional vascular finding of PA remodeling associated with hyperoxia exposure was also attenuated by treatment with lower doses of HC but unchanged in animals treated with the highest dose (Figure 2). While there was a trend toward increasing medial wall thickness with increasing $\mathrm{HC}$ doses in normoxia-exposed mice, this was not statistically significant (Figure 2). Collectively, these findings might indicate that steroids at higher doses have isolated effects on the RV, independent of other vascular effects. The underlying causes and implications of these RV alterations

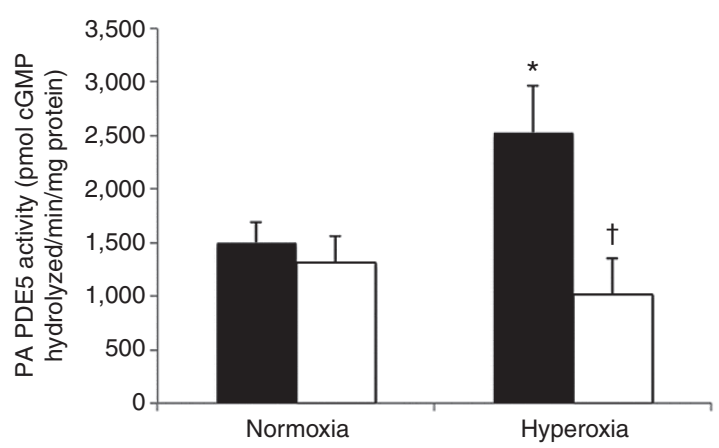

b

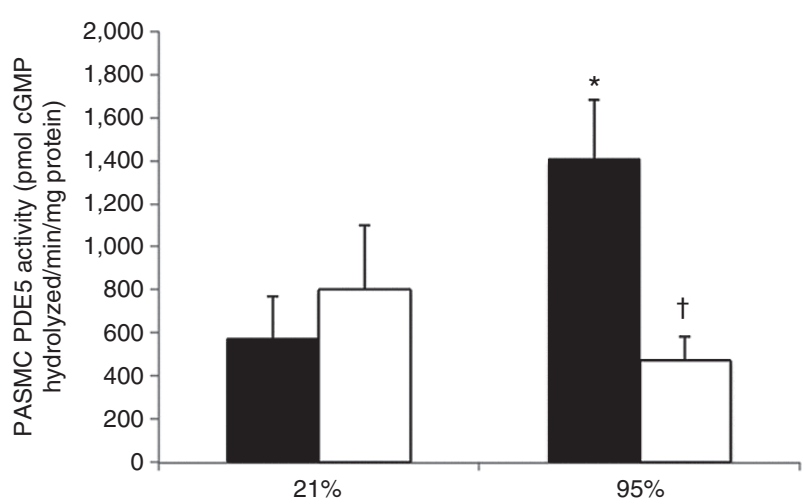

Figure 7. Hydrocortisone treatment attenuates PDE5 activation induced by hyperoxia exposure in neonatal mice and in isolated PASMC. (a) Mice exposed to $75 \% \mathrm{O}_{2}$ (hyperoxia) or room air (normoxia) \pm hydrocortisone $(1,5$, or $10 \mathrm{mg} \mathrm{HC}$ ) or sterile water (vehicle) were killed and PA protein was harvested. PDE5 specific activity was measured as the sildenafil-inhibitable fraction of total cGMP hydrolysis and normalized for total milligram of protein ( $n=5-9$ per group, black columns: vehicle, white: $1 \mathrm{mg} \mathrm{HC}$ ). Data are shown as mean $\pm \mathrm{SEM},{ }^{*} P<0.05$ vs. normoxia vehicle, ${ }^{\dagger} P<0.05$ vs. hyperoxia vehicle. (b) PASMC isolated from healthy $14-d$ neonatal mice were exposed to $95 \% \mathrm{O}_{2}-5 \% \mathrm{CO}_{2}$ or $21 \% \mathrm{O}_{2}-5 \% \mathrm{CO}$, for $24 \mathrm{~h}$ and total protein was harvested. PDE5 specific activity was measured as the sildenafil-inhibitable fraction of total cGMP hydrolysis and normalized for total milligram of protein ( $n=5-7$ per group; black columns: untreated, white: $100 \mathrm{nmol} / \mathrm{I} \mathrm{HC)}$. Data are shown as mean $\pm \mathrm{SEM},{ }^{*} P<0.05$ vs. $21 \%$ untreated, ${ }^{\dagger} P<0.05$ vs. $95 \%$ untreated. PDE5, phosphodiesterase type 5; PASMC, pulmonary artery smooth muscle cells; HC, hydrocortisone; cGMP, cyclic guanosine 59-monophosphate. 


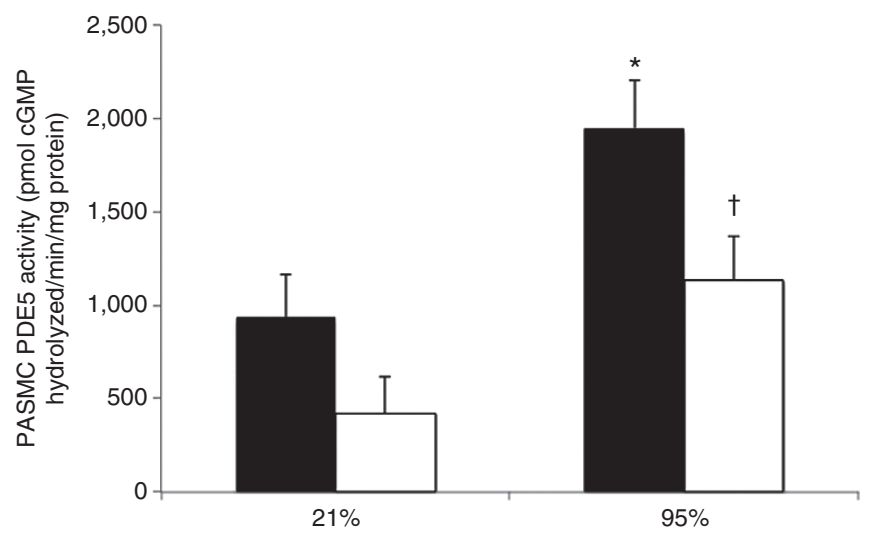

Figure 8. Hydrocortisone attenuation of hyperoxia-induced PDE5 activity is rapid. PASMC isolated from healthy $14-d$ neonatal mice were exposed to $95 \% \mathrm{O}_{2}-5 \% \mathrm{CO}_{2}$ or $21 \% \mathrm{O}_{2}-5 \% \mathrm{CO}_{2}$ for $90 \mathrm{~min}$ and total protein was harvested. PDE5 specific activity was measured as the sildenafil-inhibitable fraction of total cGMP hydrolysis and normalized for total milligram of protein ( $n=4-11$ per group; black columns: untreated, white: $100 \mathrm{nmol} / \mathrm{I}$ HC). Data are shown as mean \pm SEM, ${ }^{*} P<0.05$ vs. $21 \%$ untreated, ${ }^{t} P<0.05$ vs. $95 \%$ untreated. PDE5, phosphodiesterase type 5; PASMC, pulmonary artery smooth muscle cells; HC, hydrocortisone; CGMP, cyclic guanosine 59-monophosphate.

in otherwise healthy animals receiving glucocorticoids are not yet clear and deserve further investigation.

In addition to aberrant cardiovascular remodeling, BPD is also characterized by arrested alveolar growth and alveolar simplification $(30,31)$. This was evidenced in our model as demonstrated by increased mean alveolar area and chord length in animals exposed to chronic hyperoxia (Figure 3) (24). Glucocorticoids alone have been known to adversely affect alveolar growth, with $\mathrm{HC}$ being reported as less detrimental compared with dexamethasone $(22,32)$. In our study, we observed dose-dependent effects of $\mathrm{HC}$ on alveolar growth with worsening alveolar simplification associated with increasing $\mathrm{HC}$ doses during hyperoxia exposure (Figures 3 and 4). This effect was not seen, however, at the lowest HC dose used in these studies (Figures 3 and 4). Under normoxic conditions, only the $10 \mathrm{mg} / \mathrm{kg}$ dose of $\mathrm{HC}$ was associated with increased alveolar simplification (Figures 3 and 4 ).

To investigate potential mechanisms through which steroids impair alveolar development, we sought out to determine the effects of hyperoxia and HC treatment on parenchymal elastin content. Elastin plays an essential role in distal lung development, however, the effects of hyperoxia on elastin are not clear, with some investigators reporting that hyperoxia exposure leads to decreased lung elastin (33), with other groups reporting no differences (34). Similarly, steroids have been reported to either increase or have no effects on lung elastin $(35,36)$. We found no differences in parenchymal elastin content between animals exposed to either normoxia or hyperoxia, and no effects of steroid treatment on lung elastin (Figure 5). The exact pathways involved in the dose-dependent steroid impairment of alveolar development in our model remain unclear and deserve further investigation.
Given concerns about effects of glucocorticoid treatment on somatic growth, we also examined effects of $\mathrm{HC}$ treatment on body weight. Postnatal growth failure is a common side-effect seen in infants receiving prolonged courses of dexamethasone (37). Similar effects on body weight have been reported in rodents treated with dexamethasone (32). In addition, chronic hyperoxia exposure of neonatal mice has also been associated with poor weight gain $(23,24)$. In the current study, we did not observe any adverse effects of oxygen exposure on body weight, findings that might potentially be explained by the stress of injections affecting the growth of normoxia-exposed animals. HC treatment decreased somatic growth in two groups: normoxic animals receiving $1 \mathrm{mg} / \mathrm{kg} \mathrm{HC}$ and hyperoxic mice treated with $10 \mathrm{mg} / \mathrm{kg} \mathrm{HC}$ (Figure 6).

The exact mechanisms contributing to development of $\mathrm{PH}$ in BPD remain unclear. One potential pathway contributing to vasoconstriction is activation of PDE5. PDE5 is the primary phosphodiesterase in the lung vasculature and contributes to vasoconstriction by degrading cyclic GMP (cGMP). Our lab has previously demonstrated increased PDE5 activity in the murine model of hyperoxic lung injury $(10,38)$. We also demonstrated that hyperoxia-induced RVH and PA remodeling can be partially reversed with PDE5 inhibitor, sildenafil, underscoring the importance of aberrant cGMP signaling in the development of PH in this model (10). In order to determine whether the vascular effects of glucocorticoids seen in our model are associated with attenuation of PDE5, we investigated PDE5 activity under in vivo and in vitro following HC treatment. Consistent with our previous findings, chronic hyperoxia exposure of neonatal mice resulted in increased PA PDE5 activity (Figure 7a) (10). $1 \mathrm{mg} / \mathrm{kg}$ of HC, the lowest dose used in these studies, was sufficient to attenuate PDE5 activation (Figure 7a). In isolated mouse's PASMC, treatment with $100 \mathrm{nmol} / \mathrm{l} \mathrm{HC}$ normalized PDE5 activity induced by 24-h exposure to $95 \% \mathrm{O}_{2}$ (Figure 7b). Of note, the dose of $\mathrm{HC}$ used in in vitro experiments is equivalent to levels achieved after physiologic replacement dosing in neonates, suggesting that relatively low doses of $\mathrm{HC}$ are sufficient to attenuate aberrant PDE5 activation (39). The mechanisms underlying these effects of glucocorticoids on PDE5 are currently unknown. However, we speculate that these effects are nongenomic in nature, as demonstrated by rapid attenuation of PDE5 activity with $\mathrm{HC}$ treatment during $90 \mathrm{~min}$ of hyperoxia exposure (Figure 8). In addition, we have previously shown that PDE5 is activated under conditions of increased oxidative stress (25). Based on our previous findings that $\mathrm{HC}$ decreases markers of oxidative stress in the sheep model of persistent pulmonary hypertension of the newborn $(20,21)$, we speculate that HC impacts PDE5 activity via similar pathways in the murine model. Further studies are needed to determine the precise mechanisms of HC attenuation of PDE5 activity and whether these $\mathrm{HC}$ effects are mediated by the glucocorticoid receptor.

In conclusion, we have shown that HC treatment of hyperoxia-exposed neonatal mice decreases pulmonary vascular remodeling. We speculate that these effects might be due, at least in part, to attenuation of hyperoxia-induced PDE5 
activity. These findings provide new insights into the effects of glucocorticoids on the developing neonatal pulmonary vasculature. These findings are novel as these are the first studies of $\mathrm{HC}$ in a model of $\mathrm{PH}$ with accompanying lung disease. We acknowledge that lack of hemodynamic and long-term follow up data is a limitation of the current study. Further studies will be necessary to determine cardiovascular effects of neonatal steroid treatment on myocardial and alveolar development as the mice move toward adulthood as well as to determine the smallest dose of glucocorticoid that results in attenuation of hyperoxia-induced PDE5 activity, while avoiding aberrant effects on alveolar development.

\section{METHODS}

\section{Animal Protocols}

This study was approved by the Institutional Animal Care and Use Committee at Northwestern University. Aged-matched C57Bl/6 mice (Charles River, Wilmington, MA)\#8232; were placed in room air (normoxia) or $75 \% \mathrm{O}_{2}$ (hyperoxia) in a Plexiglass chamber (Biospherix, Lacona, NY) within $24 \mathrm{~h}$ of birth $(10,40)$. Dams were rotated every $24 \mathrm{~h}$ between normoxia and hyperoxia cages to prevent toxicity. Pups received one of three doses of $\mathrm{HC}(1,5$, or $10 \mathrm{mg} / \mathrm{kg}$ ) (Pfizer, New York, NY) subcutaneously every other day or equivalent volume of vehicle (sterile water) for $14 \mathrm{~d}$. The pups were killed after $14 \mathrm{~d}$ of exposure.

\section{Measurement of RVH}

Mouse hearts were dissected to separate the RV from the left ventricle plus septum. Fulton's Index (RV weight divided by left ventricle plus septum weight) was used to assess RVH (40).

\section{Measurement of Medial Wall Thickness}

Mouse lungs were inflation fixed at $25 \mathrm{~cm} \mathrm{H}_{2} \mathrm{O}$ with $4 \%$ formalin, stained with hematoxylin and eosin, and imaged using an Olympus BX40 microscope (40x). Six to eight images per animal were taken and analyzed in a blinded fashion. Medial wall thickness was measured as the ratio of the area of small PA wall over the total PA area $(40,41)$.

\section{Measurement of Alveolar Area}

Lung sections were stained with hematoxylin overnight, and lung morphometry images were taken with an Olympus BX40 microscope $(20 \times)$. Six to eight nonoverlapping images per animal were taken and analyzed in a blinded fashion. Mean alveolar area was measured using Scion software (Scion Corporation, Frederick, MD).

\section{Quantification of Elastin Content}

Lung sections were stained for elastin with Verhoeff-van Gieson stain (Polysciences, Warrington, PA) and imaged using an Olympus BX40 microscope (40x). Six to eight nonoverlapping images per animal were obtained and analyzed in a blinded fashion. Elastin was quantified with the NIH ImageJ program and calculated as percentage of elastin area/percentage tissue area.

\section{PA Protein Isolation}

PA protein was isolated using an iron particle infusion (10). As previously described, iron particle-containing solution was infused into the $\mathrm{RV}$, resulting in lodging of the small iron particles $(0.2 \mu \mathrm{m})$ into the small PAs (42). The lungs were then inflated with $1 \% \mathrm{w} / \mathrm{v}$ of agarose, chilled in cold phosphate buffered saline, and minced. After treatment with collagenase $(80 \mathrm{U} / \mathrm{ml})$ and manual dissociation, the vessels were lysed in $1 \times \mathrm{Mg}$ lysis buffer (Upstate, Charlottesville, VA) containing a protease inhibitor cocktail (Sigma, St. Louis, MO) and a phosphatase inhibitor cocktail (EMD Biosciences, San Diego, CA), followed by sonication. Released iron particles were removed with a Dynal magnetic particle concentrator (Invitrogen, Grand Island, NY).

\section{Cell Culture}

Primary cultures of PASMCs from small PAs of normoxic 14-d mice were prepared as previously described (43). Briefly, a slurry of $0.5 \%$ $(\mathrm{w} / \mathrm{v})$ agarose $+0.5 \%$ iron particles in M199 media was infused into the pulmonary vascular bed via the RV, resulting in lodging of 0.2 $\mu \mathrm{mol} / \mathrm{l}$ iron particles in small PAs. The lungs were then inflated with agarose, removed, and dissociated. The iron-containing vessels were pulled down with a magnet, treated with collagenase $(80 \mathrm{U} / \mathrm{ml})$, and dissociated. The resulting PASMC cultures were maintained in M199 media with $20 \%$ fetal bovine serum and antibiotics (M199 complete media) at $37{ }^{\circ} \mathrm{C}$ in a humidified atmosphere with $5 \% \mathrm{CO}_{2}-95 \%$ air. All experiments were performed using cells between passages 2 and 4. PASMC were placed in serum-free media and exposed to 21 or $95 \% \mathrm{O}_{2} \pm 100 \mathrm{nmol} / \mathrm{l} \mathrm{HC}$ (Sigma) (diluted in serum-free media) for $24 \mathrm{~h}$. Additional PASMC cultures were placed in serum-free media and exposed to 21 or $95 \% \mathrm{O}_{2} \pm 100 \mathrm{nmol} / \mathrm{l} \mathrm{HC}$ (Sigma) for $90 \mathrm{~min}$. PASMC were harvested for analysis using $1 \times \mathrm{Mg}$ lysis buffer (Upstate, New Hartford, NY) supplemented with a protease inhibitor cocktail (Sigma) and a phosphatase inhibitor cocktail (EMD Biosciences, Billerica, MA). Cell extracts were sonicated and protein concentration was determined using the Bradford assay (44).

\section{PDE5 Activity Assay}

PA protein was assayed for cGMP hydrolytic activity using a commercially available kit (Enzo, Farmingdale, NY) as previously described (25). Briefly, PA protein was purified over a Centri Spin 10 column to remove any free phosphates (Princeton Separations, Adelphia, NJ). Purified protein concentrations were determined using the Bradford method (44). Samples were assayed in duplicate for cGMP hydrolytic activity using a commercially available colorimetric cyclic nucleotide phosphodiesterase assay kit (Enzo) with and without sildenafil (100 nmol/l, Sigma-Aldrich, St. Louis, MO). Results are shown as PDE5specific pmol cGMP hydrolyzed/minute/mg total protein.

\section{Statistical Analysis}

All data are expressed as means \pm SEM, with " $n$ " representing the number of animals in each group. Significance was set at $P<0.05$. Differences between groups were analyzed by ANOVA with post-hoc Bonferroni's analysis using Prism software (Graphpad Software, San Diego, CA).

\section{STATEMENT OF FINANCIAL SUPPORT}

This work was supported by the Department of Pediatrics Physician-Scientist Research Award from Lurie Children's Hospital (M. Perez) and NIH HL109478 (K.N. Farrow).

Disclosure: The authors have no financial relationships relevant to this article to disclose.

\section{REFERENCES}

1. Fanaroff AA, Stoll BJ, Wright LL, et al.; NICHD Neonatal Research Network. Trends in neonatal morbidity and mortality for very low birthweight infants. Am J Obstet Gynecol 2007;196:147.e1-e8.

2. McAleese KA, Knapp MA, Rhodes TT. Financial and emotional cost of bronchopulmonary dysplasia. Clin Pediatr (Phila) 1993;32:393-400.

3. Stoll BJ, Hansen NI, Bell EF, et al.; Eunice Kennedy Shriver National Institute of Child Health and Human Development Neonatal Research Network. Neonatal outcomes of extremely preterm infants from the NICHD Neonatal Research Network. Pediatrics 2010;126:443-56.

4. An HS, Bae EJ, Kim GB, et al. Pulmonary hypertension in preterm infants with bronchopulmonary dysplasia. Korean Circ J 2010;40:131-6.

5. Check J, Gotteiner N, Liu X, et al. Fetal growth restriction and pulmonary hypertension in premature infants with bronchopulmonary dysplasia. J Perinatol 2013;33:553-7.

6. Khemani E, McElhinney DB, Rhein L, et al. Pulmonary artery hypertension in formerly premature infants with bronchopulmonary dysplasia: clinical features and outcomes in the surfactant era. Pediatrics 2007;120:1260-9.

7. Mourani PM, Sontag MK, Younoszai A, et al. Early pulmonary vascular disease in preterm infants at risk for bronchopulmonary dysplasia. Am J Respir Crit Care Med 2015;191:87-95.

8. Bhat R, Salas AA, Foster C, Carlo WA, Ambalavanan N. Prospective analysis of pulmonary hypertension in extremely low birth weight infants. Pediatrics 2012;129:e682-9. 


\section{Steroid effects on pulmonary vasculature}

9. Amy RW, Bowes D, Burri PH, Haines J, Thurlbeck WM. Postnatal growth of the mouse lung. J Anat 1977;124:131-51.

10. Lee KJ, Berkelhamer SK, Kim GA, et al. Disrupted pulmonary artery cyclic guanosine monophosphate signaling in mice with hyperoxia-induced pulmonary hypertension. Am J Respir Cell Mol Biol 2014;50:369-78.

11. Coalson JJ. Pathology of bronchopulmonary dysplasia. Semin Perinatol 2006;30:179-84.

12. Hislop AA, Haworth SG. Pulmonary vascular damage and the development of cor pulmonale following hyaline membrane disease. Pediatr Pulmonol 1990;9:152-61.

13. Bhatt-Mehta V, Donn SM. Sildenafil for pulmonary hypertension complicating bronchopulmonary dysplasia. Expert Rev Clin Pharmacol 2014;7:393-5.

14. Mourani PM, Sontag MK, Ivy DD, Abman SH. Effects of long-term sildenafil treatment for pulmonary hypertension in infants with chronic lung disease. J Pediatr 2009;154:379-84, 384.e1-2.

15. Maxey DM, Ivy DD, Ogawa MT, Feinstein JA. Food and Drug Administration (FDA) postmarket reported side effects and adverse events associated with pulmonary hypertension therapy in pediatric patients. Pediatr Cardiol 2013;34:1628-36.

16. Truffert P, Empana JP, Bréart G, et al. Treatment strategies for bronchopulmonary dysplasia with postnatal corticosteroids in Europe: the EURAIL survey. Acta Paediatr 2003;92:948-51.

17. da Costa DE, Nair AK, Pai MG, Al Khusaiby SM. Steroids in full term infants with respiratory failure and pulmonary hypertension due to meconium aspiration syndrome. Eur J Pediatr 2001;160:150-3.

18. Soukka H, Halkola L, Aho H, Rautanen M, Kero P, Kääpä P. Methylprednisolone attenuates the pulmonary hypertensive response in porcine meconium aspiration. Pediatr Res 1997;42:145-50.

19. Konduri GG, Bakhutashvili I, Eis A, Afolayan A. Antenatal betamethasone improves postnatal transition in late preterm lambs with persistent pulmonary hypertension of the newborn. Pediatr Res 2013;73:621-9.

20. Perez M, Lakshminrusimha S, Wedgwood S, et al. Hydrocortisone normalizes oxygenation and cGMP regulation in lambs with persistent pulmonary hypertension of the newborn. Am J Physiol Lung Cell Mol Physiol 2012;302:L595-603.

21. Perez M, Wedgwood S, Lakshminrusimha S, Farrow KN, Steinhorn RH. Hydrocortisone normalizes phosphodiesterase- 5 activity in pulmonary artery smooth muscle cells from lambs with persistent pulmonary hypertension of the newborn. Pulm Circ 2014;4:71-81.

22. Lee HJ, Kim BI, Choi ES, et al. Effects of postnatal dexamethasone or hydrocortisone in a rat model of antenatal lipopolysaccharide and neonatal hyperoxia exposure. J Korean Med Sci 2012;27:395-401.

23. Felszeghy K, Bagdy G, Nyakas C. Blunted pituitary-adrenocortical stress response in adult rats following neonatal dexamethasone treatment. J Neuroendocrinol 2000;12:1014-21.

24. Warner BB, Stuart LA, Papes RA, WispéJR. Functional and pathological effects of prolonged hyperoxia in neonatal mice. Am J Physiol 1998;275:L110-7.

25. Farrow KN, Groh BS, Schumacker PT, et al. Hyperoxia increases phosphodiesterase 5 expression and activity in ovine fetal pulmonary artery smooth muscle cells. Circ Res 2008;102:226-33.

26. Farrow KN, Lakshminrusimha S, Czech L, et al. SOD and inhaled nitric oxide normalize phosphodiesterase 5 expression and activity in neonatal lambs with persistent pulmonary hypertension. Am J Physiol Lung Cell Mol Physiol 2010;299:L109-16.

27. Israel BA, Sherman FS, Guthrie RD. Hypertrophic cardiomyopathy associated with dexamethasone therapy for chronic lung disease in preterm infants. Am J Perinatol 1993;10:307-10.

28. Zecca E, Papacci P, Maggio L, et al. Cardiac adverse effects of early dexamethasone treatment in preterm infants: a randomized clinical trial. J Clin Pharmacol 2001;41:1075-81.

29. Sicard RE, Werner JC. Dexamethasone induces a transient relative cardiomegaly in neonatal rats. Pediatr Res 1992;31:359-63.

30. Stenmark KR, Abman SH. Lung vascular development: implications for the pathogenesis of bronchopulmonary dysplasia. Annu Rev Physiol 2005;67:623-61.

31. Thébaud B, Abman SH. Bronchopulmonary dysplasia: where have all the vessels gone? Roles of angiogenic growth factors in chronic lung disease. Am J Respir Crit Care Med 2007;175:978-85.

32. Fayon M, Jouvencel P, Carles D, Choukroun ML, Marthan R. Differential effect of dexamethasone and hydrocortisone on alveolar growth in rat pups. Pediatr Pulmonol 2002;33:443-8.

33. Bruce MC, Pawlowski R, Tomashefski JF Jr. Changes in lung elastic fiber structure and concentration associated with hyperoxic exposure in the developing rat lung. Am Rev Respir Dis 1989;140:1067-74.

34. Ramani M, Bradley WE, Dell'Italia LJ, Ambalavanan N. Early exposure to hyperoxia or hypoxia adversely impacts cardiopulmonary development. Am J Respir Cell Mol Biol 2015;52:594-602.

35. Kovar J, Willet KE, Hislop A, Sly PD. Impact of postnatal glucocorticoids on early lung development. J Appl Physiol (1985) 2005;98:881-8.

36. Loehle M, Schwab M, Kadner S, et al. Dose-response effects of betamethasone on maturation of the fetal sheep lung. Am J Obstet Gynecol 2010;202:186.e1-7.

37. Doyle LW, Ehrenkranz RA, Halliday HL. Early ( $<8$ days) postnatal corticosteroids for preventing chronic lung disease in preterm infants. Cochrane Database Syst Rev 2014;5:CD001146.

38. Heilman RP, Lagoski MB, Lee KJ, et al. Right ventricular cyclic nucleotide signaling is decreased in hyperoxia-induced pulmonary hypertension in neonatal mice. Am J Physiol Heart Circ Physiol 2015;308:H1575-82.

39. Watterberg KL, Scott SM. Evidence of early adrenal insufficiency in babies who develop bronchopulmonary dysplasia. Pediatrics 1995;95:120-5.

40. Aslam M, Baveja R, Liang OD, et al. Bone marrow stromal cells attenuate lung injury in a murine model of neonatal chronic lung disease. Am J Respir Crit Care Med 2009;180:1122-30.

41. Ladha F, Bonnet S, Eaton F, Hashimoto K, Korbutt G, Thébaud B. Sildenafil improves alveolar growth and pulmonary hypertension in hyperoxiainduced lung injury. Am J Respir Crit Care Med 2005;172:750-6.

42. Farrow KN, Lee KJ, Perez M, et al. Brief hyperoxia increases mitochondrial oxidation and increases phosphodiesterase 5 activity in fetal pulmonary artery smooth muscle cells. Antioxid Redox Signal 2012;17: 460-70.

43. Lee KJ, Czech L, Waypa GB, Farrow KN. Isolation of pulmonary artery smooth muscle cells from neonatal mice. J Vis Exp 2013:e50889.

44. Bradford MM. A rapid and sensitive method for the quantitation of microgram quantities of protein utilizing the principle of protein-dye binding. Anal Biochem 1976;72:248-54. 